\title{
La función mediadora de la capacidad absortiva en la relación entre el capital intelectual y la innovación en los institutos de educación superior del sur de Tamaulipas \\ The mediating function of absorptive capacity in the relationship between intellectual capital and innovation in higher education institutes in southern Tamaulipas
}

\author{
Bernardo Nahuat-Román ${ }^{1}$ (i) 0000-0003-1291-0880 \\ Miriam Rodríguez-Vargas 1 (c) 0000-0002-4243-0370 \\ Ma del Carmen Gómez de la Fuente ${ }^{1}$ (C) 0000-0003-0062-617X \\ ${ }^{1}$ Universidad Autónoma de Tamaulipas $\$ bernienahuat@gmail.com \\ (C) Universidad De La Salle Bajío (México)
}

Palabras clave: capital intelectual, capacidad absortiva, innovación organizacional, educación superior, universidad, stakeholders, capital relacional, capital humano, transferencia de conocimiento, mediación, ecuaciones estructurales, mínimos cuadrados parciales

Keywords: intellectual capital, absorptive capacity, organizational innovation, higher education, university, stakeholders, relational capital, human capital, knowledge transfer, mediation, structural equation modeling, partial least squares

Recepción: 16 - 02 - 2021 / Aceptación: 26 - 05 - 2021

\section{Resumen}

Introducción: en la actualidad, la innovación en las organizaciones es demandada de una forma sin precedentes. Si bien el capital intelectual (CI) ha sido señalado como uno de sus detonadores, un paso adelante es entender el rol que tienen otras variables en esta relación. Por lo anterior, el objetivo principal de la presente investigación es determinar la función mediadora de la capacidad absortiva (CA) en la relación entre el CI y la innovación organizacional en institutos de educación superior del sur de Tamaulipas.

Método: de los 31 institutos de educación superior existentes en el sur de Tamaulipas se seleccionaron aquellos que tienen menos de 50 empleados, reduciéndose a 27 escuelas. En estas instituciones se aplicaron 92 cuestionarios a docentes en el primer semestre del año 2020 para, posteriormente, aplicar un modelo de ecuaciones estructurales con la técnica PLS.

Resultados: la evidencia confirma la función mediadora de la CA en la relación entre el CI y la innovación organizacional. Así mismo, los resultados permiten observar un efecto positivo y significativo de las variables CI y CA sobre la innovación organizacional. 
La función mediadora de la capacidad absortiva en la relación entre el capital intelectual y la innovación en los institutos de educación superior del sur de Tamaulipas

Discusión: el resultado amplía el conocimiento de la función mediadora que tiene la CA en la relación entre el CI y la innovación organizacional; de la misma forma, sugiere que, en la medida en que se pueda controlar este efecto de la CA, se podría favorecer la innovación.

\section{Abstract}

Introduction: at present, innovation in organizations is demanded in an unprecedented way. Although intellectual capital (CI) has been identified as one of its triggers, one step forward is to understand the role that other variables have in this relationship. Therefore, the main objective of this research is to determine the mediating function of absorptive capacity (CA) in the relationship between $\mathrm{CI}$ and organizational innovation in higher education institutes in southern Tamaulipas.

Method: from the 31 existing higher education institutes in southern Tamaulipas, those with less than 50 employees were selected, reducing to 27 schools. In this institutions, 92 teacher questionnaires were applicated in the first semester of 2020 to later apply a structural equations model with the PLS technique.

Results: the evidence confirms the mediating role of CA in the relationship between $\mathrm{CI}$ and organizational innovation. Likewise, the results allow to observe a positive and significant effect of the $\mathrm{CI}$ and $\mathrm{CA}$ variables on organizational innovation.

Discussion: the result broadens the knowledge of the mediating function that $\mathrm{CA}$ has in the relationship between $\mathrm{CI}$ and organizational innovation, in the same way it suggests that to the extent that this effect of CA can be controlled, innovation could be favored.

\section{$\underline{\text { Introducción }}$}

En un mundo caracterizado por una feroz competencia, sumergido en la globalización y con una clara tendencia al conocimiento (Teece y Pisano, 1994) la innovación en las organizaciones se convierte en un factor clave para la sobrevivencia, dejando de ser una opción para convertirse en una necesidad (Buenechea-Elberdin, 2017; Li y Yu, 2018). Diversos autores han relacionado la innovación con factores clave de las organizaciones, como su crecimiento (Koudelková y Milichovsky, 2015), su éxito y la ventaja competitiva que puedan desarrollar (Varadarajan y Jayachandran, 1999), por lo que se requiere profundizar tanto en sus detonadores como en la forma como estos interactúan entre sí. 
En este sentido, el CI a través de aproximaciones empíricas previas ha demostrado que tiene un efecto positivo y significativo sobre los distintos tipos de innovaciones que se encuentran en la literatura (por ejemplo: Agostini y Nosella, 2017; Al-Jinini, Dahiyat y Bontis, 2019; Tsou, Chen y Liao, 2016), llevándolo a ser señalado como generador de innovación (Nejjari y Aamoum, 2020). Autores como Cano, Sanchez, González y Pérez (2014) son radicales al señalar que difícilmente una organización puede generar innovación sin contemplar al CI como una parte fundamental.

La CA por su parte también ha sido estrechamente relacionada con la innovación (CepedaCarrion, Cegarra-Navarro y Jimenez-Jimenez, 2012) y reconocida por mejorar el desempeño innovador de las organizaciones (Zahra y George, 2002) lo cual es lógico considerando que en ella recae la habilidad de las organizaciones para reconocer y adquirir conocimiento para posteriormente aplicarlo con fines comerciales (Cohen y Levinthal, 1990).

Si bien la influencia del CI sobre la innovación se ha abordado previamente en la literatura un paso adelante es determinar la función mediadora que cumplen otras variables en esta relación, por ello, esta investigación se propone como objetivo determinar la función mediadora que tiene la CA en la relación entre el CI y la innovación. Según Engelman, Fracasso y Schmidt (2017) son pocos los trabajos que han abordado la relación entre el CI y la CA lo cual se confirma con la revisión de la literatura realizada reduciéndose más a aquellos que han abordado la función mediadora de la CA en la relación entre el CI y la innovación (por ejemplo: Cassol, Gonçalo y Ruas, 2016; Kousar, Zafar, Batool y Sajjad, 2019; Soo, Wei Tian, Teo y Cordery, 2017).

Por otro lado, con la llegada de la pandemia generada por el COVID 19 los institutos de educación superior como parte del sector de la educación se han visto forzados a generar una serie de cambios radicales en múltiples sentidos. En un corto tiempo, desde la comunicación con sus alumnos hasta la forma de enseñanza sufrieron innovaciones que les están permitiendo adaptarse y continuar cumpliendo con sus funciones principales por lo que el presente estudio puede significar una luz en la presente situación.

En concreto, considerando la escasa literatura de la función mediadora de la CA en la relación entre el CI e innovación y sobre todo la importancia de profundizar en la innovación en los institutos de educación superior, este trabajo se fija como objetivo principal determinar la función mediadora de la capacidad absortiva (CA) en la relación entre el capital intelectual (CI) y la innovación organizacional en institutos de educación superior del sur de Tamaulipas. Como 
La función mediadora de la capacidad absortiva en la relación entre el capital intelectual y la innovación en los institutos de educación superior del sur de Tamaulipas

objetivo complementario, se propone determinar el efecto que tienen el CI y la CA sobre la innovación organizacional.

La presente investigación está estructurada en cinco secciones. La primera termina con esta breve introducción. En la segunda sección se presenta una revisión de la literatura de CI, CA, innovación organizacional e institutos de educación superior para, posteriormente, en la siguiente sección abordar la metodología que se siguió para llevar a cabo el estudio. En la cuarta sección se presentan los resultados obtenidos y, finalmente, en la última sección se cierra con las conclusiones, las limitaciones y futuras líneas de investigación.

\section{Revisión de la literatura}

\section{Capital intelectual}

El CI puede entenderse de forma muy generalizada como los activos intangibles que la empresa posee o a los que tiene acceso (Buenechea-Elberdin, 2017; Gomezelj y Smolčić, 2016). Aunque existe quienes afirman que sus orígenes se remontan a principios de la humanidad (Edvinson y Malone, 1999), su estudio real inició a partir de los años noventa, con autores pioneros que buscaban medirlo con diferentes metodologías (por ejemplo: Brooking, 1997; Kaplan y Norton, 1997; Saint-Onge, 1996; Sveiby, 1997). Probablemente, el estudio más emblemático sea el de Leif Edvinsson con el modelo Navigator (Edvinsson, 1997).

La importancia del CI se pone de manifiesto cuando autores clásicos como Roos, Bainbridge y Jacobsen (2001) señalan que el éxito de una organización está relacionado en un 80 $\%$ con sus activos intangibles mientras que los activos tangibles únicamente influyen en un $20 \%$. Kousar et al. (2019) coinciden sobre la importancia de los activos intangibles llevando al CI como tema central en los debates sobre desarrollo económico y específicamente en el contexto de las universidades como un factor relevante (Chatterji y Kiran, 2017).

Aunque no existe una definición consensuada, la literatura parece coincidir en que se trata de un concepto multidimensional (Engelman et al., 2017) integrado por los llamados capitales humano, estructural y relacional siendo el primero de estos el principal (Nejjari y Aamoum, 2020). Los 3 capitales, aunque estrechamente correlacionados tienen raíces distintas, el capital humano proviene del conocimiento, habilidades y experiencias de los empleados, el capital estructural se refiere al conocimiento encriptado en las rutinas de la organización y el capital relacional se refiere a las relaciones que tiene la organización con sus stakeholders (Agostini, Nosella y Filippini, 2017).

No 27, Vol. 14 (1), 2021. ISSN 2007 - 0705, pp.: 1 - 28

$$
-4 \text { - }
$$


El CI al provenir de los recursos internos de las organizaciones y ser constantemente señalado como factor de ventaja competitiva (Ansari, Barati y Sharabiani, 2016; Delgado-Verde, Martín-De Castro y Amores-Salvadó, 2016) es considerado por autores como Buenechea-Elberdin (2017) parte de la teoría basada en los recursos (Barney, 1991; Wernerfelt, 1984). En el caso de la presente investigación el desarrollo del marco teórico fue inspirado desde esta perspectiva.

Por otro lado, al ser básicamente conocimiento (Chen, Zhao y Wang, 2015; Delgado-Verde, Martín De Castro, Navas-López y Amores-Salvadó, 2014) genera un interés natural por determinar cómo interactúa con otras variables también basadas en el conocimiento como es la capacidad absortiva que tienen las organizaciones. En este sentido, pocos estudios han abordado de forma simultánea ambas variables en su carácter de variables independientes con la innovación.

\section{Capacidad absortiva}

El origen de la CA parte de la propuesta realizada por Cohen y Levinthal (1989) a finales de los ochentas, si bien sus autores la conceptualizaron es un hecho que en la comunidad científica todavía no está consensuada (Duchek, 2013; Lane, Koka y Pathak, 2006). Así pues, para la presente investigación se entiende como la habilidad de una organización para de forma permanente absorber, diseminar y utilizar nuevo conocimiento orientado hacia una ventaja competitiva sostenida (Soo et al., 2017).

La CA nace de la incapacidad de generar el conocimiento interno necesario en las organizaciones para ser competitivas requiriendo adquirirlo de otras, ese conocimiento adquirido posteriormente se incorpora al existente y se convierte en una nueva base de conocimientos más enriquecida que la existente de forma previa (Flatten, Engelen, Zahra y Brettel, 2011; Spithoven, Clarysse y Knockaert, 2011).

Ahora bien, se trata de un concepto multidimensional integrado por un conjunto de rutinas y procesos en las organizaciones que juntas componen una capacidad dinámica organizacional basada en 4 componentes: Adquisición, asimilación, transformación y explotación de conocimiento (Zahra y George, 2002). Siguiendo con los mismos autores, adquisición se refiere a la capacidad de recuperar, identificar y adquirir conocimiento externo. Asimilación de conocimientos se relaciona con la capacidad de la empresa para analizar, procesar, interpretar y comprender la información obtenida de fuentes externas. Por su parte, la transformación se refiere al reconocimiento de 2 grupos de información aparentemente incongruentes y su combinación para 
La función mediadora de la capacidad absortiva en la relación entre el capital intelectual y la innovación en los institutos de educación superior del sur de Tamaulipas

alcanzar un nuevo esquema. Por último, la explotación del conocimiento está relacionada con la capacidad de las organizaciones para refinar, expandir y apalancar y crear habilidades existentes y encaminadas en la aplicación del conocimiento (Cohen y Levinthal, 1990; Zahra y George, 2002).

Aunque diversos trabajos han evidenciado su influencia en variables claves como la innovación (Tsai, 2001), las finanzas (Kostopoulos, Papalexandris, Papachroni y Loannou, 2011), la transferencia de conocimiento (Szulanski, 1996), el aprendizaje (Lane, Salk y Lyles, 2001), la mayoría de las investigaciones son de carácter teórico (Solís, Zerón y Sánchez, 2019) generando una laguna en el conocimiento. En este sentido, Lewin, Massini y Peeters (2011, p.81) señalan que "las rutinas y procesos organizacionales específicos que constituyen las capacidades de capacidad de absorción siguen siendo una caja negra”.

La función de la CA resulta clave para la innovación organizacional como argumentan Zahra y George (2002) y como se ha comprobado con trabajos empíricos llevados a cabo recientemente (por ejemplo: Solís et al., 2019); sin embargo, al tener las organizaciones diferente capacidad de absorción del conocimiento trae como consecuencia que cada empresa genere una capacidad para innovar diferente (Subramaniam y Youndt, 2005) surgiendo dudas que requieren ser contestadas.

\section{Innovación organizacional}

La innovación es uno de los factores críticos en los resultados de una organización (Iqbal, Latif, Marimon, Sahibzada y Hussain, 2018) al grado de depender su sobrevivencia de su capacidad para innovar (Li y Yu, 2018). Diversos autores la consideran pieza clave en la generación de ventaja competitiva (Shahsiah y Sepahvand, 2016) cubriendo con ella las nuevas necesidades que demandan sus clientes. En la actualidad ha adquirido un mayor valor por la competencia sin precedentes que se vive (Patky y Pandey, 2020) motivada en gran parte por la globalización.

Su conceptualización puede ser definida en distintas formas (Alshamsi, Isaac y Bhaumik, 2019a), por ejemplo, Rogers (2003) la asocia con una idea, práctica u objeto que es percibido como nuevo por un individuo u otra unidad de adopción, Nelson y Winter (1977) la entienden como la iniciación, adopción e implementación de ideas o actividades nuevas adoptadas en la organización y para Alrowwad, Abualoush y Masa'deh (2020, p. 201) “es un proceso que identifica oportunidades para producir nuevos servicios o productos. La innovación es la adopción de nuevas 
ideas y conocimientos para desarrollar y mejorar nuevos productos". Dando como constantes en las definiciones propuestas las palabras idea y nuevo.

Ahora bien, Schumpeter (1934) ha sido uno de los autores que más ha influido tanto en teorías, conceptualizaciones y clasificaciones existentes rechazando teorías clásicas y neoclásicas (Huang, Leone, Caporuscio y Kraus, 2020) y dando como resultado que diversos organismos sigan su huella al momento de abordar y conceptualizar la innovación (por ejemplo: OCDE, 2005). Las clasificaciones de la innovación en la literatura son abundantes, por ejemplo, se puede mencionar la innovación incremental y radical (Afuah, 1998), la innovación abierta (Chesbrough, 2003), las innovaciones de productos, procesos, mercadotecnia y organizativas (OCDE, 2005), entre otras.

Debido a que en la literatura los términos desempeño de la innovación, innovación organizacional e innovación empresarial se usan indistintamente (Chang, Gong, Way y Jia, 2013) este trabajo entiende la innovación organizacional relacionada con el sector educativo como aquellas modificaciones que se enfocan en cubrir las necesidades cambiantes de los alumnos, basándose en una cultura organizacional donde el recurso humano es clave y está pensada en la sustentabilidad. Respecto al alcance de la innovación se puede entender como algo completamente nuevo para el mercado o nuevo únicamente para la organización que la implementa (SantosRodrigues, Fernández-Jardón y Figueroa, 2015); el segundo criterio es el que aplica para el presente estudio.

\section{Los institutos de educación superior}

Los institutos de educación superior (entiéndase universidades y tecnológicos) de acuerdo a Herrera y Suárez (2021, p. 149) tienen 3 funciones primordiales: "la investigación, motor de la ciencia y el pensamiento humanístico, que mediante procesos epistemológicos logra innovar conocimiento básico y aplicado en las distintas ramas de la actividad científica. En segunda instancia, la docencia como espacio privilegiado para el intercambio y el libre debate de las ideas; es un mecanismo para transmitir el conocimiento y generar procesos de enseñanza-aprendizaje en la comunidad estudiantil. Y, por último, la extensión, la cual hace posible que la universidad se conecte con la sociedad y contribuya al progreso económico y social a través de la vinculación con los demás actores del sistema".

Desempeñan así mismo un rol fundamental en el desarrollo social y económico de los países, tanto desarrollados como en vías de desarrollo (Iqbal, Latif, Marimon, Sahibzada y Hussain, 
La función mediadora de la capacidad absortiva en la relación entre el capital intelectual y la innovación en los institutos de educación superior del sur de Tamaulipas

2018). Herrera y Suárez (2021) los identifican como agentes de cambio en sus áreas de influencia mientras que Kousar et al. (2019) apunta que dependen en buena medida de la innovación para conservar su permanencia en el mercado.

Ahora bien, los institutos de educación superior están inmersos en un contexto complejo que incluye la reducción de apoyos estatales, el cambio en las formas de gobernanza, el cambio de modelos de aprendizaje, presiones de responsabilidad hacia la sociedad, entre otras (Chatterji y Kiran, 2017) obligándolas a cambiar su enfoque de lo tangible a lo intangible buscando con ello sobrevivir y desarrollarse (Kousar et al., 2019).

Considerando que a decir de Kousar et al. (2019, p. 659) los institutos de educación superior "son responsables de producir conocimientos, habilidades y relaciones entre los seres humanos; por lo que es un sector ideal para estudiar la relación entre capital intelectual e innovación organizacional" y principalmente por el reto que enfrentan para desempeñar sus funciones en época de la pandemia generada por el COVID 19 esta investigación los adopta como unidad de análisis.

\section{Planteamiento de hipótesis}

Engelman et al. (2017) en su estudio empírico realizado en 495 empresas manufactureras en Brasil, aplicaron un cuestionario medido con una escala Likert de 7 puntos a directores, presidentes, administradores de recursos humanos y de investigación y desarrollo para posteriormente tratar los datos con ecuaciones estructurales basadas en el análisis de la covarianza y comprobar que el CI influye sobre la CA llevando a las empresas a ser más o menos innovadoras y también que la CA tiene una influencia positiva y significativa sobre el desempeño de la innovación.

Cassol et al. (2016) también en Brasil, pero mediante un estudio de caso en una empresa dedicada al papel y cartón recabaron 104 cuestionarios de gerentes, coordinadores y supervisores para posteriormente aplicar ecuaciones estructurales con la técnica PLS y comprobar que existen relaciones positivas entre el capital intelectual, la capacidad absortiva y la innovación y que el rol mediador de la CA es capaz de potencializar el CI y fomentar la innovación. Los autores complementaron su estudio con técnicas cualitativas como son la observación y entrevistas semiestructuradas.

En su estudio realizado en Pakistán, Kousar, Zafar, Batool y Sajjad (2019) incluyen como componente del CI al capital espiritual para investigar el papel mediador de la CA en la relación entre el CI y la innovación organizacional, tomando como unidad de análisis institutos de 
educación superior tanto públicos como privados recaban 610 cuestionarios y en base a un modelo de ecuaciones estructurales basado en la covarianza comprobaron que la capacidad absortiva tiene un rol mediador entre el capital intelectual y la innovación organizacional, que el CI y la CA tienen una relación positiva y significativa con la innovación organizacional y que el CI y la CA tienen una relación positiva entre sí. Con base en lo anterior se proponen las siguientes cuatro hipótesis:

H1. El capital intelectual tiene un efecto positivo y significativo en la innovación organizacional. H2. El capital intelectual tiene un efecto positivo y significativo en la capacidad absortiva.

H3. La capacidad absortiva tiene un efecto positivo y significativo en la innovación organizacional. H4. La capacidad absortiva tiene una función mediadora entre el capital intelectual y la innovación organizacional. La fig. 1 presenta las relaciones propuestas a comprobar.

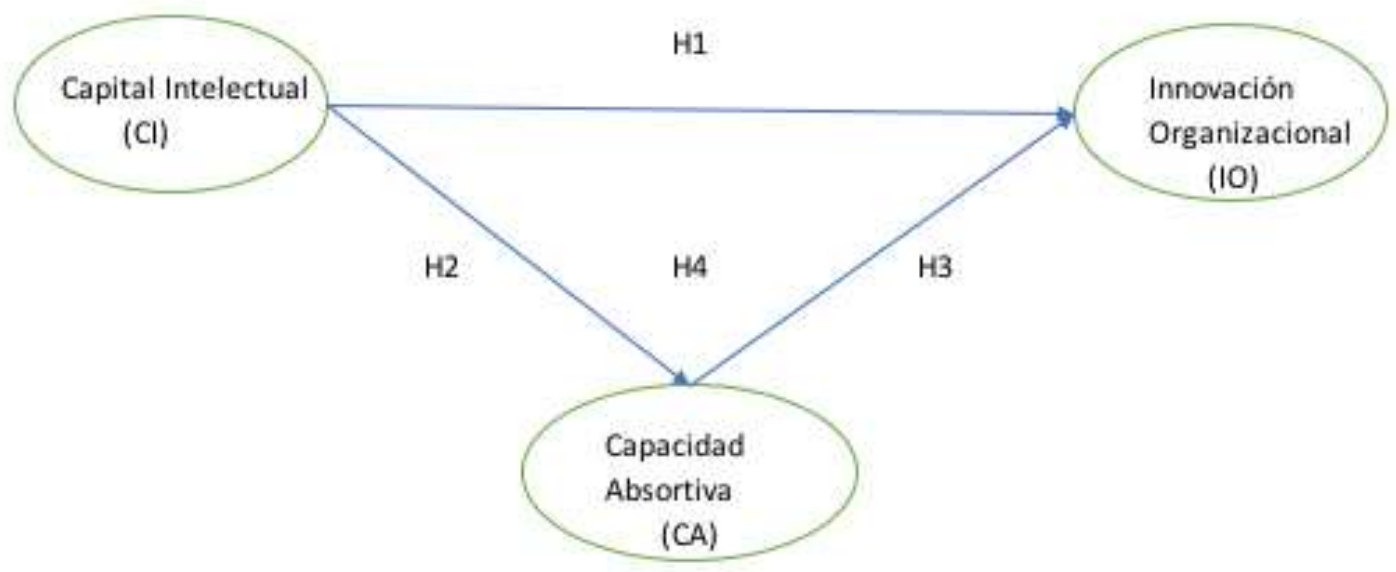

Fig. 1. Modelo conceptual propuesto de investigación.

Fuente: elaboración propia.

Fig. 1. Proposed conceptual research model.

Source: own elaboration.

\section{Método}

Para lograr el objetivo de la presente investigación, se adoptó un enfoque cuantitativo de corte transversal y con alcance explicativo. A continuación, se describe la metodología seguida.

\section{Muestra y unidad de análisis}

Como unidad de análisis se consideró el total (censo) de las instituciones de educación superior (universidades y tecnológicos), tanto públicas como privadas en los municipios de Tampico, 
La función mediadora de la capacidad absortiva en la relación entre el capital intelectual y la innovación en los institutos de educación superior del sur de Tamaulipas

Madero y Altamira, en el estado de Tamaulipas. Esto de acuerdo con el Directorio Estadístico Nacional de Unidades Económicas (DENUE), generado por el Instituto Nacional de Estadística y Geografía (INEGI) y disponible en línea (Instituto Nacional de Estadística y Geografía, 2020).

Se eligieron estos municipios por la diversidad de oferta escolar que presentan y por agrupar la mayor concentración de población en el estado. En el caso de las escuelas que tienen diversos campus o facultades se consideró cada una de manera independiente tal como lo genera el DENUE.

Siguiendo algunos trabajos previos de CI e Innovación en otros contextos (Delgado-Verde et al., 2016; Sánchez-Baez, Fernández-Serrano y Romero, 2018), se aplicó como filtro que las instituciones seleccionadas tuvieran más de 50 empleados, buscando con ello que exista un flujo de conocimientos más dinámico. Así pues, un total de 27 institutos (14 públicos y 13 privados) fueron finalmente considerados como unidad de análisis para el presente estudio.

\section{Instrumento de recolección de datos}

Al ser el CI un constructo multidimensional reflejado en los capitales humano, estructural y relacional se hace necesario manejarlo como un constructo de segundo orden. En lo referente a la CA, buscando aportar al conocimiento se sigue a Cassol et al. (2016), quien lo manejó como un constructo de segundo orden integrado por las siguientes dimensiones: capacidad absortiva realizada y capacidad absortiva potencial. A decir del autor "La CA se puede subdividir en dos dimensiones: CA potencial y CA realizada. La CA potencial puede considerarse como un bagaje de conocimientos que ha sido adquirido y asimilado, pero que permanece en estado latente hasta que surge un uso real del conocimiento; a su vez, la CA realizada se preocupa por la transformación y aplicación de los conocimientos previamente adquiridos para lograr una ventaja competitiva a desarrollar en el corto o mediano plazo" (Cassol et al., 2016, p. 5). Por último la variable innovación organizacional se maneja como un constructo de primer orden.

Así pues, el CI al ser un constructo de segundo orden, consideró inicialmente todos los reactivos de sus 3 dimensiones, 15 en total. Cada una de sus dimensiones (constructos de primer orden) contaron con sus reactivos específicos: cinco para la dimensión capital humano (Engelman et al., 2017); cinco para la dimensión de capital estructural (Engelman et al., 2017); y para la dimensión capital relacional también cinco reactivos (Cassol et al., 2016).

La CA al ser también un constructo de segundo orden estuvo compuesta por los 12 ítems de sus 2 dimensiones, es decir, seis reactivos de la dimensión capacidad absortiva potencial 
(Cassol et al., 2016; Engelman et al., 2017), y seis reactivos de la dimensión capacidad absortiva realizada (Cassol et al., 2016; Engelman et al., 2017; Soo et al., 2017).

Por último, la variable innovación organizacional se manejó como un constructo de primer orden con cinco ítems (Alshamsi et al., 2019a; Cassol et al., 2016).

Así pues, se construyó un cuestionario específico y especialmente para esta investigación, que incluyó 32 ítems tomados de la literatura existente, con base en percepciones y adaptados para el sector de la educación (ver tabla 1). Para su medición, se utilizó una escala Likert de siete puntos, donde 1 significa totalmente en desacuerdo, hasta llegar a 7, que significa totalmente de acuerdo. Atendiendo a la literatura, todos los reactivos fueron manejados como reflectivos.

Como informantes clave se eligió a cuatro personas de cada instituto que contaran con la siguiente característica: docentes con antigüedad menor a 1 año, buscando con ello recabar lo que se vive en el interior de cada instituto. En este sentido, se incluyeron en el estudio ocho docentes que estaban próximos a cumplir un año de labores en un plazo menor a un mes, entendiendo que esto no distorsionaría los resultados a obtener y sí robustecería el modelo. Si bien en la literatura no existe un consenso respecto al tamaño de la muestra para aplicar un modelo de ecuaciones estructurales (Hoyle y Kenny, 1999) es necesario adquirir el suficiente poder estadístico para lograr generalizar los resultados (Kousar et al., 2019). 
La función mediadora de la capacidad absortiva en la relación entre el capital intelectual y la innovación en los institutos de educación superior del sur de Tamaulipas

Tabla 1. Detalles de medidas.

Table 1. Details of measures.

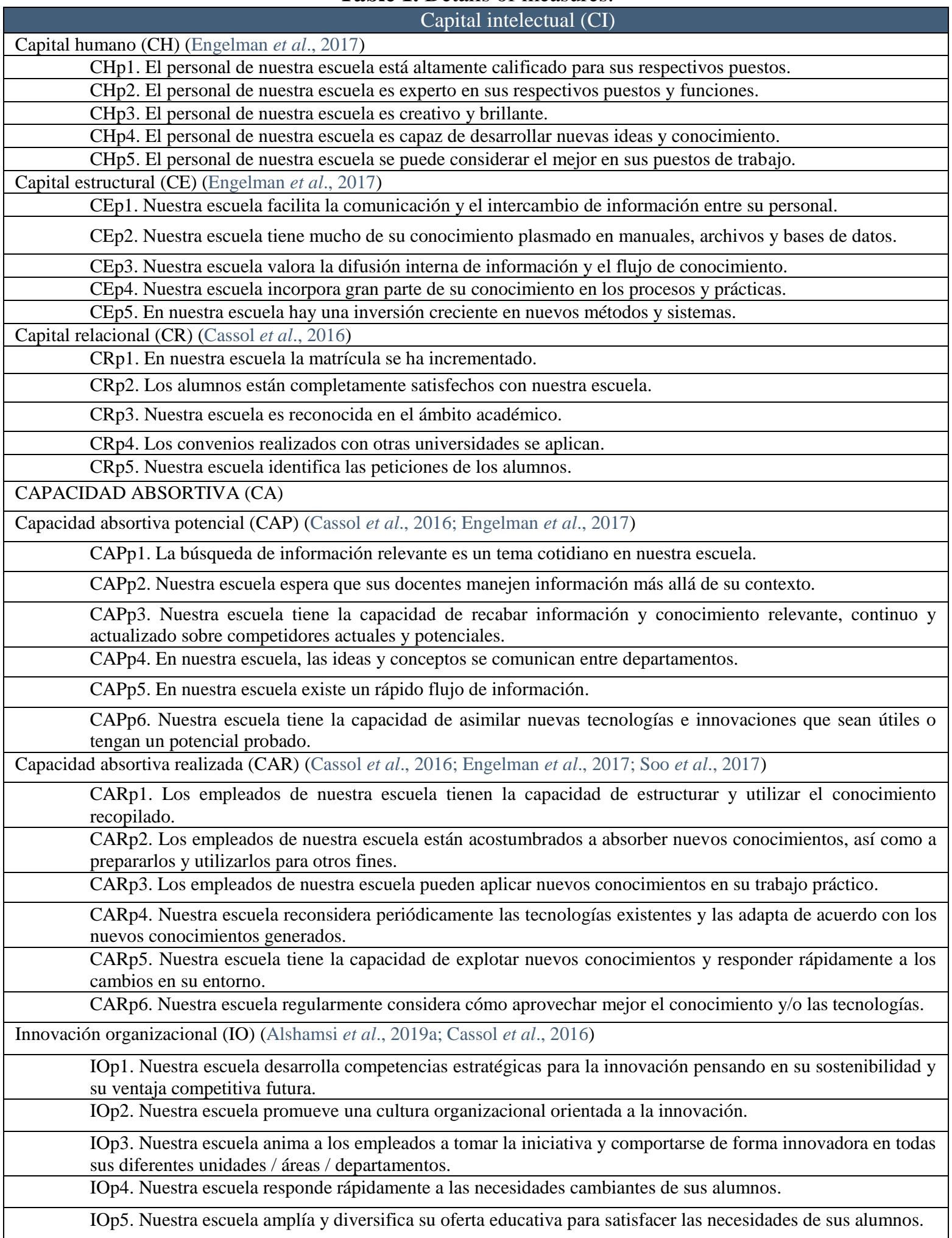

Fuente: elaboración propia.

Source: own elaboration.

No 27, Vol. 14 (1), 2021. ISSN 2007 - 0705, pp.: 1 - 28

-12 - 


\section{Técnica utilizada}

Las observaciones recabadas fueron tratadas mediante un modelo de ecuaciones estructurales con la técnica PLS (siglas de Partial Least Squares), el cual es un enfoque basado en la varianza (Wold, 1985), específicamente con el software Smartpls versión 3.2.8 (Ringle, Wende y Becker, 2015). Esta técnica, junto con este software, gozan de gran popularidad en el estudio de la relación CIInnovación en el contexto de la educación (Iqbal et al., 2018; Shahtaheri y Teymournejad, 2020) y, entre sus características está que no exige una distribución normal de los datos (Hair, Hult, Ringle y Sarstedt, 2017), y puede trabajar con muestras pequeñas (Asiaei, Jusoh y Bontis, 2018).

Respecto a la comprobación del tamaño de la muestra, aunque la técnica no establece premisas rigurosas (Martínez y Fierro, 2018), algunos autores mencionan que las recomendaciones mínimas están entre 30 y 100 casos (Cepeda y Roldán, 2004). Otra forma de validar el tamaño de la muestra es siguiendo la regla de Barclay, Higgins y Thompson (1995) que señala que la muestra requerida estará en función de la regresión múltiple más compleja que se pueda encontrar requiriendo 10 observaciones por cada una, cumpliendo por mucho las 92 observaciones recabadas en el presente estudio.

Para determinar el efecto mediador de la CA en la relación entre el CI y la innovación organizacional, se utilizó la prueba de SOBEL (Sobel Test Calculator for the Significance of Mediation) que, a decir de Baron y Kenny (1986), es utilizada para medir la fuerza del efecto indirecto que ejerce una variable independiente sobre una dependiente, teniendo como hipótesis nula que el efecto es igual a cero.

\section{Procedimiento}

La encuesta se llevó a cabo en el primer semestre del año 2020 de forma presencial y vía correo electrónico, garantizando confidencialidad y anonimato a los encuestados. De los 104 cuestionarios que originalmente se pensaban recabar, un total de 92 cuestionarios utilizables fueron obtenidos y tratados inicialmente con el software Statistical Package for the Social Sciences (SPSS por sus siglas en inglés) no encontrándose con valores incongruentes que pudieran alterar los resultados del estudio y procediendo a generar estadística descriptiva (ver tabla 2). Para facilitar su tratamiento, los reactivos fueron codificados poniendo, primero, la inicial del constructo que las agrupa y asignándoles un número de pregunta. Por ejemplo, $\mathrm{CHp} 1$ se refiere a la pregunta 1 del 
La función mediadora de la capacidad absortiva en la relación entre el capital intelectual y la innovación en los institutos de educación superior del sur de Tamaulipas

constructo de primer orden capital humano; CAPp3 significa la pregunta 3 del constructo de primer orden capacidad absortiva potencial, y así sucesivamente para todos.

Tabla 2. Características de los encuestados

Table 2. Characteristics of the respondents

\begin{tabular}{|ccc|}
\hline Característica & Observaciones & Porcentaje \\
\hline Sexo & & \\
Hombre & 43 & $46 \%$ \\
Mujer & 49 & $54 \%$ \\
Total & 92 & $100 \%$ \\
Posgrado & & \\
Si & 74 & $80 \%$ \\
No & 18 & $20 \%$ \\
Total & 92 & $100 \%$ \\
Antigüedad en la escuela & & \\
Más de 1 año & 8 & $42 \%$ \\
Menos de 1 año & 39 & $50 \%$ \\
Menos de 5 años & 45 & $100 \%$ \\
Total & 92 & \\
\hline
\end{tabular}

Fuente: elaboración propia con base en SPSS.

Source: own elaboration based on SPSS.

El análisis en PLS se realizó en 2 etapas, en la primera de ellas, la valoración del modelo de medida (también llamado modelo externo) se revisó que las cargas de los ítems cumplieran con los mínimos exigidos, que se cumpliera la fiabilidad de los constructos mediante el Alfa de Cronbach y la fiabilidad compuesta y también se comprobó la validez convergente mediante la varianza media extraída (average variance extracted o AVE por sus siglas en inglés).

La segunda etapa se refiere al ajuste del modelo SEM, también llamado estructural o interno. En esta etapa se analiza la evaluación de los coeficientes path y la valoración de las $\mathrm{R}^{2}$ permitiendo validar las hipótesis 1,2 y 3 .

\section{$\underline{\text { Resultados }}$}

\section{Ajuste del modelo de medida}

Un primer paso en el análisis de los datos fue la prueba kolmogorov-Smirnov, como se puede ver en la tabla 3 los p valor fueron menores a $<0.01$, por lo que se rechaza la hipótesis nula de manera altamente significativa, lo que permite afirmar que no se presenta normalidad en los datos recabados y se confirma la elección del método PLS. Para iniciar con el modelo de medida, se procedió a replicar todos los ítems de los constructos de primer orden en sus respectivos constructos 
de segundo orden (Hair et al., 2017). Entiéndase por constructos a las variables y a los ítems como sinónimos de reactivos. Posteriormente se depuraron aquellos ítems que no cumplían con el 0.7 exigido por la literatura (Hair et al., 2017). En el caso de la depuración de ítems se depura simultáneamente en los constructos de segundo orden. En total, 6 ítems fueron eliminados en esta etapa (CHp5, CRp5,CARp1,CARp2, CARp3, CAPp3), restando para el presente trabajo un total de 26, como se puede ver en la tabla 5.

Tabla 3.Prueba de Kolmogorov-Smirnov.

Table 3. Kolmogorov-Smirnov test.

\begin{tabular}{|ccc|c|}
\hline Variable & Estadístico & gl & $\mathrm{P}$ \\
\hline $\mathrm{CH}$ & .142 & 92 & 0.000 \\
$\mathrm{CE}$ & .216 & 92 & 0.000 \\
$\mathrm{CR}$ & .134 & 92 & 0.000 \\
CAR & .166 & 92 & 0.000 \\
CAP & .137 & 92 & 0.000 \\
IO & .168 & 92 & 0.000 \\
\hline
\end{tabular}

Fuente: elaboración propia con base en SPSS.

Source: own elaboration based on SPSS.

En lo referente a la validez discriminante que evalúa el grado en que las variables son independientes entre sí (Hair et al., 2017) se utilizó el criterio de Fornell-Larcker (Fornell y Larcker, 1981) que compara las raíces cuadradas de los valores de la varianza promedio extraída (AVE). En la tabla 4 se puede ver que los valores de la AVE de todos los constructos eran más altos que sus correlaciones con otros constructos (Henseler, Ringle y Sinkovics, 2009). En este sentido, "para una validez discriminante adecuada, los elementos diagonales deben ser mayores que los elementos fuera de la diagonal en las filas y columnas correspondientes, y exceder 0.5" (Mura y Longo, 2013, p. 443) lo cual indica una adecuada validez discriminante entre constructos.

Tabla 4. Matriz de correlaciones de acuerdo al criterio de Fornell-Larcker para constructos de primer orden.

Table 4. Correlation matrix according to the Fornell-Larcker criterion for first order constructs.

\begin{tabular}{|c|c|c|c|c|c|c|}
\hline & CAP & CAR & CE & CH & CR & IO \\
\hline CAP & $\mathbf{0 . 7 7 9}$ & & & & & \\
\hline CAR & 0.887 & $\mathbf{0 . 9 0 6}$ & & & & \\
\hline CE & 0.499 & 0.416 & $\mathbf{0 . 8 9 3}$ & & & \\
\hline CH & 0.670 & 0.584 & 0.813 & $\mathbf{0 . 8 4 8}$ & & \\
\hline CR & 0.718 & 0.687 & 0.585 & 0.777 & $\mathbf{0 . 8 5 1}$ & \\
\hline IO & 0.908 & 0.870 & 0.521 & 0.737 & 0.797 & $\mathbf{0 . 8 8 6}$ \\
\hline
\end{tabular}

Fuente: elaboración propia.

Source: own elaboration. 
La función mediadora de la capacidad absortiva en la relación entre el capital intelectual y la innovación en los institutos de educación superior del sur de Tamaulipas

Para validar los criterios de calidad de los constructos, es decir, la consistencia interna se utilizó el Alfa de Cronbach y la confiabilidad compuesta. Estos indicadores permiten definir qué parte de la medición obtenida por la escala es atribuible al constructo que se desea medir y cuanto proviene de fluctuaciones casuales (Bollen, 1989). en la tabla 5 puede observarse que todos los constructos superaron el 0.70 recomendado (Hair et al., 2017) aunque existen autores que hablan de 0.80 para considerarse una buena consistencia interna (Mura y Longo, 2013).

La validez convergente refleja el grado en que una escala se correlaciona de forma positiva con otras medidas del mismo constructo (Malhotra, 2012) llegando a ser el ideal aquella donde los valores del AVE son $\geq 0.50$ lo cual da la certeza que la variable latente explica más de la mitad de la varianza de sus indicadores (Tabachnick y Fidell, 2001). En este caso los valores obtenidos son superiores a 0.5 cumpliendo por lo tanto con lo sugerido por la literatura (Nunally y Bernstein, 1994).

Tabla 5. Validación de la confiabilidad de constructos de primer y segundo orden.

Table 5. Validation of the reliability of first and second order constructs.

\begin{tabular}{|c|c|c|c|c|c|}
\hline & \multicolumn{3}{|c|}{ Validez convergente } & \multicolumn{2}{c|}{$\begin{array}{c}\text { Criterios de calidad } \\
\text { Consistencia interna }\end{array}$} \\
\hline $\begin{array}{c}\text { Constructos } \\
\text { de segundo } \\
\text { orden }\end{array}$ & $\begin{array}{c}\text { Constructos } \\
\text { de primer } \\
\text { orden }\end{array}$ & $\begin{array}{c}\text { Número de ítems } \\
\text { (después de la } \\
\text { depuración) } \\
>0.70\end{array}$ & AVE & $\begin{array}{c}\text { Confiabilidad } \\
\text { compuesta }\end{array}$ & $\begin{array}{c}\text { Alfa de } \\
\text { Cronbach }\end{array}$ \\
\hline CA & & 8 & 0.589 & 0.919 & $>0.70$ \\
\hline & CAP & 5 & 0.607 & 0.885 & 0.837 \\
\hline CI & CAR & 3 & 0.650 & 0.847 & 0.725 \\
\hline & CH & 4 & 0.611 & 0.953 & 0.946 \\
\hline & CE & 5 & 0.720 & 0.911 & 0.870 \\
\hline & CR & 4 & 0.798 & 0.952 & 0.936 \\
\hline & IO & 5 & 0.725 & 0.913 & 0.870 \\
\hline
\end{tabular}

Fuente: elaboración propia.

Source: own elaboration.

\section{Valoración del modelo estructural}

El modelo estructural, también llamado, SEM o interno fue validado primeramente con los coeficientes de determinación $\left(\mathrm{R}^{2}\right)$ los cuales indican la calidad del modelo ajustado ya que muestran una medida de la proporción de variación en los constructos endógenos explicados por el modelo SEM. En este sentido, Cohen (1988) señala que una $\mathrm{R}^{2}$ igual a un $2 \%$ indica un efecto 
pequeño, del $13 \%$ al $25 \%$ puede considerarse como un efecto mediano y una $\mathrm{R}^{2} \geq 26 \%$ indica un efecto grande. Lo anterior, de acuerdo a los resultados obtenidos le da una buena calificación al presente modelo y sugiere una correcta revisión previa de la literatura.

La fig. 2 resume el resultado obtenido. La variable innovación organizacional con una $\mathrm{R}^{2}$ de 0.876 fue explicada en gran porcentaje por las variables independientes capital intelectual y capacidad absortiva, aunque definitivamente la segunda de estas presenta un mayor poder explicativo. Un punto relevante surge al observarse que el efecto del CI en la IO es pequeño, mientras el efecto del CI en la CA es grande; esto sugiere, en una primera instancia, que el CI requiere de la variable CA como vehículo para potencializar su efecto en la IO.

El resultado coincide con lo encontrado por Cassol et al. (2016) lo que permite ser más concluyente en los resultados e inferir que las variables capital intelectual y capacidad absortiva son detonadoras de la innovación organizacional. En general, el modelo tiene amplio poder explicativo de todos los constructos endógenos mostrando $\mathrm{R}^{2}$ aceptables, además los coeficientes path son positivos y significativos en las relaciones propuestas (ver fig. 2) lo cual permite aceptar hasta aquí las H1, H2 y H3.

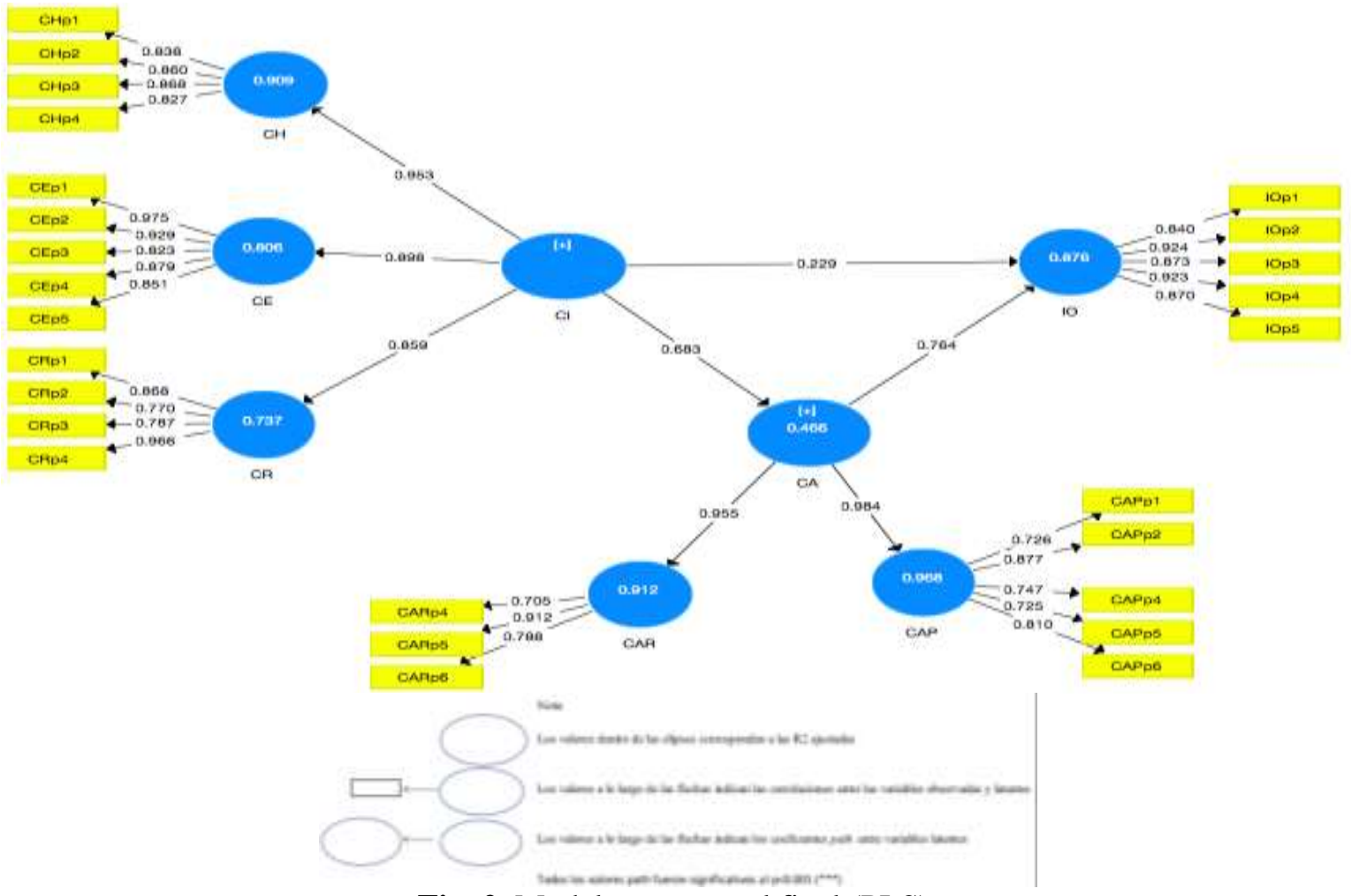

Fig. 2. Modelo estructural final (PLS).

Fuente: elaboración propia.

Fig. 2. Final structural model (PLS).

Source: own elaboration. 
La función mediadora de la capacidad absortiva en la relación entre el capital intelectual y la innovación en los institutos de educación superior del sur de Tamaulipas

La H4 proponía que la capacidad absortiva tiene una función mediadora en la relación entre el CI y la innovación organizacional para lo cual se utilizó la prueba SOBEL (Sobel Test Calculator for the Significance of Mediation) disponible en línea (Free Statistics Calculators, 2020) y así poder determinar si el mediador cumple esa función. En estudios de gestión que buscan comprobar los efectos de una mediación única el test de Sobel determina la fuerza del efecto indirecto que tiene la variable exógena sobre la variable endógena tomando como hipótesis nula que el efecto es igual a cero (Baron y Kenny, 1986). El estadístico de Sobel sigue una distribución de prueba z, así que los valores $>1.96$ indican un $\mathrm{p}<0.05$ (Cassol et al., 2016) (ver tabla 6).

Tabla 6. Resultados de prueba de mediación de Sobel

Table 6. Sobel Mediation Test Results

\begin{tabular}{|c|c|c|c|}
\hline Variable exógena & Variable endógena & Sobel & P \\
\hline Capital intelectual & $\begin{array}{c}\text { Innovación } \\
\text { organizacional }\end{array}$ & 7.30 & 0.000 \\
\hline
\end{tabular}

Fuente: elaboración propia

Source: own elaboration.

Considerando que otros autores han comprobado sus propuestas de mediación por dos o más vías (por ejemplo: Cassol et al., 2016; Martinez-Conesa et al., 2017), el presente estudio confirma la mediación también con el enfoque de mínimos cuadrados parciales (PLS) basado en bootstrapping para SEM. Para el procedimiento propuesto por Hair et al. (2017), se midió primeramente el efecto directo (sin el mediador) del CI sobre la IO, para posteriormente determinar un efecto indirecto con el mediador incluido, dando como resultado que la CA parcialmente media el efecto del CI en la IO (ver tabla 7).

Tabla 7. Análisis de mediación con Bootstrapping PLS.

Table 7. Mediation analysis with Bootstrapping PLS.

\begin{tabular}{|c|c|c|c|c|c|}
\hline \multirow[b]{2}{*}{ Relación } & \multirow[b]{2}{*}{$\begin{array}{c}\text { Efectos } \\
\text { directos (sin } \\
\text { mediador) }\end{array}$} & \multirow[b]{2}{*}{$\begin{array}{l}\text { Efectos } \\
\text { indirectos } \\
\quad(\text { con } \\
\text { mediador })\end{array}$} & \multirow[b]{2}{*}{$\begin{array}{c}\text { Efectos } \\
\text { totales }\end{array}$} & \multicolumn{2}{|c|}{ Bootstrapping } \\
\hline & & & & Estadístico t & $\mathrm{P}$ valor \\
\hline $\mathrm{CI} \rightarrow \mathrm{IO}$ & $0.229 * * *$ & $\begin{array}{c}\text { Mediación } \\
\text { parcial }\end{array}$ & 0.522 & & \\
\hline $\mathrm{CI} \rightarrow \mathrm{CA} \rightarrow \mathrm{IO}$ & -- & -- & -- & 6.764 & 0.000 \\
\hline
\end{tabular}

Fuente: datos de investigación ingresados en software SmartPLS.

Source: research data entered into SmartPLS software. 
El resultado mostrado en las tablas 6 y 7 permite inferir que la capacidad absortiva si tiene una función mediadora en la relación entre el capital intelectual y la innovación organizacional aceptando con esto la $\mathrm{H} 4$.

\section{Discusión}

Este estudio, de acuerdo con sus resultados, comprobó el efecto mediador de la CA en la relación entre el CI y la innovación organizacional aceptando con ello la H4. Así mismo comprobó que el CI tiene un efecto positivo y significativo sobre la innovación organizacional (H1), que el CI tiene un efecto positivo y significativo sobre la $\mathrm{CA}(\mathrm{H} 2)$ y que la $\mathrm{CA}$ tiene un efecto positivo y significativo sobre la innovación organizacional (H3).

Los resultados son similares a lo encontrado en la literatura; otros autores plantearon modelos parecidos aunque con diferentes metodologías y contextos (Cassol et al., 2016; Engelman et al., 2017; Kousar et al., 2019) lo que permite ser más concluyente al afirmar que el efecto del CI sobre la innovación organizacional se puede potencializar mediante la CA.

Sobre el resultado de la H1 existe una línea de estudios que han venido abordando la relación CI e innovación (véase Buenechea-Elberdin, 2017; Nahuat y Ochoa, 2020) encontrando en la mayoría de los casos una influencia positiva y significativa del CI sobre las diferentes clasificaciones de innovación existentes en la literatura. Esta relación de causalidad se ha comprobado en contextos tan diversos como una estación de policía (Alshamsi, Isaac y Bhaumik, 2019b), en la pequeña y mediana empresa (Agostini et al., 2017), en la industria financiera y hotelera (Tsou et al., 2016), aseguradoras (Shahsiah y Sepahvand, 2016), entre otras, siendo la industria manufacturera la más repetitiva (Prester, Podrug y Darabos, 2016; Zhang, Lettice y Pawar, 2019; Zhang, Qi, Wang, Pawar y Zhao, 2018).

En lo que se refiere al resultado de la H3 que evidenció una influencia del CA sobre la innovación organizacional diversos estudios respaldan el resultado obtenido con resultados similares (por ejemplo: Huang y Rice, 2009; Solís et al., 2019) por lo que a pesar de ser un concepto relativamente nuevo se puede inferir una influencia sobre la innovación organizacional. En resumen, el resultado de la H1 y H3 coincide con lo dicho por Lo, Wang y Chen (2020), tanto la capacidad absortiva como el capital intelectual tienen un rol esencial en la innovación que generan las empresas. 
La función mediadora de la capacidad absortiva en la relación entre el capital intelectual y la innovación en los institutos de educación superior del sur de Tamaulipas

\section{Conclusiones}

La investigación cumplió con su objetivo principal, es decir, determinó y evidenció la función mediadora de la capacidad absortiva en la relación entre el capital intelectual y la innovación organizacional. Así mismo, cumplió con el objetivo complementario fijado, comprobando un efecto positivo y significativo de las variables CI y CA sobre la innovación organizacional surgiendo con ello las siguientes implicaciones:

Aunque en la actualidad, con la situación generada por la pandemia de la COVID 19, ha quedado demostrada la capacidad de innovar de los institutos de educación superior como parte del sector educativo, este tipo de estudios sugieren que existe todavía un potencial mayor, a la espera de combinar el conocimiento que fluye en ellos. Las repercusiones de identificar los impulsores de la innovación son incalculables si se considera que mucha de la competitividad que requieren las empresas nace, en gran parte, de la educación formal recibida por sus recursos humanos. En otras palabras, identificar los detonantes de la innovación en los institutos de educación representa un primer paso para elevar la competitividad en la educación, y esto traería como una consecuencia una mayor competitividad en las empresas, al integrarse los estudiantes a la vida laboral.

La CA de acuerdo a su función mediadora evidenciada se convierte en una llave de paso que regula la relación entre el CI y la innovación organizacional. Una llave de paso de la cual se sabe poco y que por la necesidad actual se requiere seguir profundizando para aprovechar todo su potencial. Otro punto por considerar es la doble función que desempeña la CA, es decir, tanto como detonadora directa de la innovación, así como mediadora de la influencia ejercida por el CI.

Respecto al objetivo complementario el resultado se alineó con el grueso de la literatura, aunque en estudios previos se habían obtenido resultados similares son pocos los trabajos que permiten comparar de manera simultánea el efecto que tienen el CI y la CA sobre la innovación organizacional. Los institutos de educación superior por la esencia de su función son piezas clave en la base productiva y en el desarrollo del país por lo que en la medida que se logre detonar la innovación se podría generar beneficios en sus stakeholders.

La presente investigación presenta dos limitantes, la primera es que durante su desarrollo se declaró la pandemia por COVID 19 lo que puede generar sesgo en los datos recabados. Otra limitante es que existen escasas aproximaciones similares reduciendo con ello la oportunidad para comparar resultados. Para futuras líneas de investigación se sugiere considerar como unidad de 
análisis las escuelas de educación media superior, lo cual permitiría construir un panorama más amplio respecto al sector de la educación del sur de Tamaulipas.

\section{$\underline{\text { Referencias }}$}

Afuah, A. (1998). Innovation Management: Strategies, Implementation, and Profits. New York: Oxford University Press.

Agostini, L., y Nosella, A. (2017). Enhancing radical innovation performance through intellectual capital components. Journal of Intellectual Capital, 18(4), 789-806. https://doi.org/10.1108/JIC-10-2016-0103

Agostini, L., Nosella, A., y Filippini, R. (2017). Does intellectual capital allow improving innovation performance? A quantitative analysis in the SME context. Journal of Intellectual Capital, 18(2), 400-418. https://doi.org/10.1108/JIC-05-2016-0056

Al-Jinini, D. K., Dahiyat, S. E., y Bontis, N. (2019). Intellectual capital, entrepreneurial orientation, and technical innovation in small and medium-sized enterprises. Knowledge and Process Management, 26(2), 69-85. https://doi.org/10.1002/kpm.1593

Alrowwad, A., Abualoush, S. H., y Masa'deh, R. (2020). Innovation and intellectual capital as intermediary variables among transformational leadership, transactional leadership, and organizational performance. Journal of Management Development, 39(2), 196-222. https://doi.org/10.1108/JMD-02-2019-0062

Alshamsi, S., Isaac, O., y Bhaumik, A. (2019a). Effect of transformational leadership on intellectual capital and organizational innovation. International Journal on Emerging Technologies, 10(1), 66-76.

Alshamsi, S., Isaac, O., y Bhaumik, A. (2019b). The effects of intellectual capital on organizational innovation within Abu Dhabi police in UAE. International Journal on Emerging Technologies, 10(1), 50-58.

Ansari, R., Barati, A., y Sharabiani, A. A. A. (2016). The role of dynamic capability in intellectual capital and innovative performance. International Journal of Innovation and Learning, 20(1), 47. https://doi.org/10.1504/IJIL.2016.076671

Asiaei, K., Jusoh, R., y Bontis, N. (2018). Intellectual capital and performance measurement systems in Iran. Journal of Intellectual Capital, 19(2), 294-320. https://doi.org/10.1108/JIC$\underline{11-2016-0125}$ 
La función mediadora de la capacidad absortiva en la relación entre el capital intelectual y la innovación en los institutos de educación superior del sur de Tamaulipas

Barclay, D., Higgins, C., y Thompson, R. (1995). The partial least squares (PLS) approach to causal modeling: personal computer adoption and use as an illustration. Technology Studies, Special Issue on Research Metodology, 2(2), 285-309.

Barney, J. (1991). Firm resources and sustained competitive advantage. Journal of Mangement, 17(1), 99-120.

Baron, R., y Kenny, D. (1986). The moderator-mediator variable distinction in social psychological research: conceptual, strategic, and statistical considerations. Journal of Personality and Social Psychology, 51(6), 1173-1182. https://doi.org/10.1037//0022-3514.51.6.1173

Bollen, K. (1989). Structural Equations with Latent Variables. New York: Wiley.

Brooking, A. (1997). El capital intelectual. El principal activo de las empresas del tercer milenio (Primera). Barcelona, España: Paidós.

Buenechea-Elberdin, M. (2017). Structured literature review about intellectual capital and innovation. Journal of Intellectual Capital, 18(2), 262-285. https://doi.org/10.1108/JIC-07$\underline{2016-0069}$

Cano, M. del C., Sanchez, G. C., González, M., y Pérez, J. (2014). El rol del capital intelectual en la innovación de las empresas. European Scientific Journal, 10(28), 348-366.

Cassol, A., Gonçalo, C. R., y Ruas, R. L. (2016). Redefining the relationship between intellectual capital and innovation: The mediating role of absorptive capacity. BAR-Brazilian Administration Review, 13(4), e150067. https://doi.org/10.1590/1807-7692bar2016150067

Cepeda-Carrion, G., Cegarra-Navarro, J. G., y Jimenez-Jimenez, D. (2012). "The effect of absorptive capacity on innovativeness: context and information systems capability as catalysts.” British Journal of Management, 23(1), 110-129.

Cepeda, G., y Roldán, J. L. (2004). Aplicando en la práctica la técnica PLS en la administración de empresas. In Congreso de la ACEDE, sep. 19, 20 y 21. Murcia, España. Retrieved from http://repositorio.unan.edu.ni/2986/1/5624.pdf

Chang, S., Gong, Y., Way, S., y Jia, L. (2013). Flexibility-oriented HRM systems, absorptive capacity, and market responsiveness and firm innovativeness. Journal of Management, 39(7), 1924-1951.

Chatterji, N., y Kiran, R. (2017). Role of human and relational capital of universities as underpinnings of a knowledge economy: A structural modelling perspective from north Indian universities. International Journal of Educational Development, 56(June), 52-61. 
https://doi.org/10.1016/j.ijedudev.2017.06.004

Chen, J., Zhao, X., y Wang, Y. (2015). A new measurement of intellectual capital and its impact on innovation performance in an open innovation paradigm. International Journal of Technology Management, 67(1), 1. https://doi.org/10.1504/IJTM.2015.065885

Chesbrough, H. (2003). Open Innovation. Boston, Massachusetts: Harvard Business School Publishing Corporation.

Cohen, J. (1988). Statistical power analysis for the behavioral sciences (2nd.). New York: [OB] $\mathrm{B}$ Psychology Press.

Cohen, W., y Levinthal, D. (1989). Innovation and learning: The two faces of R\&D. The Economic Journal, 99(397), 569-596.

Cohen, W., y Levinthal, D. (1990). Absorptive Capacity: A New Perspective-.on Learning and Innovation. Administrative Science Quarterly, 35(1), 128-152. https://doi.org/10.1016/b9780-7506-7223-8.50004-6

Delgado-Verde, M., Martín-De Castro, G., y Amores-Salvadó, J. (2016). Intellectual capital and radical innovation: Exploring the quadratic effects in technology-based manufacturing firms. Technovation, 54, 35-47. https://doi.org/10.1016/j.technovation.2016.02.002

Delgado-Verde, M., Martín De Castro, G., Navas-López, J. E., y Amores-Salvadó, J. (2014). Vertical relationships, complementarity and product innovation: An intellectual capital-based view. Knowledge Management Research and Practice, 12(2), 226-235. https://doi.org/10.1057/kmrp.2012.59

Duchek, S. (2013). Capturing absorptive capacity: A critical review and future prospects. Schmalenbach Business Review, 65(3), 312-329.

Edvinson, L., y Malone, M. S. (1999). El capital intelectual: Como identificar y calcular el valor inexplotado de los recursos intangibles de su empresa. (Gestión 2000, Ed.). España: Gestión 2000.

Edvinsson, L. (1997). Developing intellectual capital at Skandia. Long Range Planning, 30(3), 366-373. https://doi.org/10.1016/s0024-6301(97)90248-X

Engelman, R., Fracasso, E., y Schmidt, S. (2017). Intellectual capital, absorptive capacity and product innovation. Management Decision, 55(3), 474-490. https://doi.org/https://doi.org/10.1108/MD-05-2016-0315

Flatten, T. C., Engelen, A., Zahra, S. A., y Brettel, M. (2011). A measure of absorptive capacity: 
La función mediadora de la capacidad absortiva en la relación entre el capital intelectual y la innovación en los institutos de educación superior del sur de Tamaulipas

Scale development and validation. European Management Journal, 29(2), 98-116. https://doi.org/10.1016/j.emj.2010.11.002

Fornell, C., y Larcker, D. (1981). Evaluating structural equation models with unobservable variables and measurement error. American Marketing Association, 109(4), 555-562. https://doi.org/10.1177/002224378101800104

Free Statistics Calculators. (2020). Sobel Test Calculator for the significance of mediation. Retrieved June 20, 2020, from https://www.danielsoper.com/statcalc/calculator.aspx?id=31

Gomezelj, D., y Smolčić, D. (2016). The influence of intellectual capital on innovativeness and growth in tourism SMEs: empirical evidence from Slovenia and Croatia. Economic ResearchEkonomska Istraživanja, $\quad 29(1), \quad$ 1075-1090. https://doi.org/10.1080/1331677X.2016.1211946

Hair, J. F. J., Hult, G. T. M., Ringle, C. M., y Sarstedt, M. (2017). A primer on partial least squares structural equation modeling (PLS-SEM) (Segunda). Sage publications.

Henseler, J., Ringle, C. M., y Sinkovics, R. R. (2009). The use of partial least squares path modeling in international marketing. Advances in International Marketing, 20(January), 277-319. https://doi.org/10.1108/S1474-7979(2009)0000020014

Herrera, F., y Suárez, J. (2021). Rol de las universidades en el Sistema Nacional de Innovación mexicano. Revista Venezolana de Gerencia, (93), 139-157.

Hoyle, R., y Kenny, D. (1999). Sample size, reliability, and tests of statistical mediation. Statistical Strategies for Small Sample Research, 1, 195-222.

Huang, F., y Rice, J. (2009). The role of absorptive capacity in facilitating open innovation outcomes: A study of Australian SMEs in the manufacturing sector. Int. J. Innov. Manag., (13), 201-220.

Huang, H., Leone, D., Caporuscio, A., y Kraus, S. (2020). Managing intellectual capital in healthcare organizations. A conceptual proposal to promote innovation. Journal of Intellectual Capital. https://doi.org/10.1108/JIC-02-2020-0063

Instituto Nacional de Estadística y Geografía. (2020). Directorio Estadístico Nacional de Unidades Económicas.

Iqbal, A., Latif, F., Marimon, F., Sahibzada, U. F., y Hussain, S. (2018). From knowledge management to organizational performance: Modelling the mediating role of innovation and intellectual capital in higher education. Journal of Enterprise Information Management, 
32(1), 36-59. https://doi.org/10.1108/JEIM-04-2018-0083

Kaplan, R., y Norton, D. (1997). Cuadro de Mando Integral. Gestión 2000 (Segunda). Barcelona, España.

Kostopoulos, K., Papalexandris, A., Papachroni, M., y Loannou, G. (2011). Absorptive capacity, innovation, and financial performance. Journal of Business Research, 64(12), 1335-1343.

Koudelková, P., y Milichovsky, F. (2015). Successful innovation by motivation. Business: Theory and PracTice, 16(3), 223-230. https://doi.org/10.3846/btp.2015.472

Kousar, S., Zafar, M., Batool, S. A., y Sajjad, A. (2019). The mediating role of absorptive capacity in the relationship between intellectual capital and organizational innovation in higher education institutes of Punjab, Pakistan. Pakistan Journal of Commerce and Social Science, 13(3), 656-679.

Lane, P., Salk, J., y Lyles, M. (2001). Absorptive capacity, learning, and performance in international joint ventures. Strategic Management Journal, 22(12), 1139-1161.

Lane, Peter, Koka, B., y Pathak, S. (2006). The reification of absorptive capacity: A critical review and rejuvenation of the construct. Academy of Management Review, 31(4), 833-863.

Lewin, A., Massini, S., y Peeters, C. (2011). Microfoundations of internal and external absorptive capacity routines. Organization Science, 22(11), 81-98.

Li, J., y Yu, D. (2018). The path to innovation: The antecedent perspective of intellectual capital and organizational character. Frontiers in Psychology, 9(DEC), 1-18. https://doi.org/10.3389/fpsyg.2018.02445

Lo, C., Wang, C., y Chen, Y. C. (2020). The mediating role of intellectual capital in open innovation in the service industries. Sustainability (Switzerland), 12(12), 1-12. https://doi.org/10.3390/su12125220

Malhotra, N. (2012). Pesquisa de marketing: uma orientação aplicada (Sexta). Porto Alegre: Bookman.

Martinez-Conesa, I., Soto-Acosta, P., y Palacios-Manzano, M. (2017). Corporate social responsibility and its effect on innovation and firm performance: An empirical research in SMEs. Journal of Cleaner Production, 142, 2374-2383. https://doi.org/10.1016/j.jclepro.2016.11.038

Martínez, M., y Fierro, E. (2018). Aplicación de la técnica PLS-SEM en la gestión del conocimiento: un enfoque técnico práctico / Application of the PLS-SEM technique in 
La función mediadora de la capacidad absortiva en la relación entre el capital intelectual y la innovación en los institutos de educación superior del sur de Tamaulipas

Knowledge Management: a practical technical approach. RIDE Revista Iberoamericana para la Investigación y el Desarrollo Educativo (Vol. 8). https://doi.org/10.23913/ride.v8i16.336

Mura, M., y Longo, M. (2013). Developing a tool for intellectual capital assessment: An individuallevel perspective. Expert Systems, 30(5), 436-450. https://doi.org/10.1111/j.1468$\underline{0394.2012 .00650 . x}$

Nahuat, B., y Ochoa, M. L. (2020). Capital intelectual y su relación con la innovación: una revisión de la literatura. Revista de Psicología y Ciencias Del Comportamiento de La Unidad Académica de Ciencias Jurídicas y Sociales, 11(2), 196-213. https://doi.org/10.29059/rpcc.20201215-124

Nejjari, Z., y Aamoum, H. (2020). Intellectual capital as a generator of innovation in companies: A systematic review. Humanities and Social Sciences Reviews, 8(1), 464-479. https://doi.org/10.18510/hssr.2020.8158

Nelson, R., y Winter, S. (1977). In search of useful theory of innovation. Research Policy, 6(1), $33-76$.

Nunally, J., y Bernstein, I. (1994). Psychometric Theory. New York: Mc Graw-Hill.

OCDE. (2005). Oslo Manual. OECD y Eurostat. https://doi.org/10.1787/9789264013100-en

Patky, J., y Pandey, S. K. (2020). Does Flexibility in Human Resource Practices Increase Innovation? Mediating Role of Intellectual Capital. South Asian Journal of Human Resources Management, 7(2), 257-275. https://doi.org/10.1177/2322093720934243

Prester, J., Podrug, N., y Darabos, M. (2016). Four-Component Model of Intellectual Capital and its Impact on Process and Product Innovations. In Proceedings of the 8th European conference on Intellectual Capital ECIC 2016. Academic Conferences and Publishing International Limited Reading.

Ringle, C., Wende, S., y Becker, J. (2015). SmartPLS 3. http://www.smartpls.com.

Rogers, E. (2003). Difussion of innovations (Third Edit). Free Press, New York.

Roos, G., Bainbridge, A., y Jacobsen, K. (2001). Intellectual capital analysis as a strategic tool. Strategy \& Leadership, 29(4), 21-26. https://doi.org/10.1108/10878570110400116

Saint-Onge, H. (1996). Tacit knowledge the key to the strategic alignment of intellectual capital. Strategy \& Leadership, 24(2), 10-16.

Sanchez-Baez, E., Fernandez-Serrano, J., y Romero, I. (2018). Personal values and entrepreneurial attitude as intellectual capital: impact on innovation in small enterprises. Amfiteatru 
Economic, 20(4), 771-787. https://doi.org/10.24818/EA/2018/49/771

Santos-Rodrigues, H., Fernandez-Jardón, C. M., y Figueroa, P. (2015). Relation between intellectual capital and the product process innovation. International Journal of KnowledgeBased Development, 6(1), 15-33. https://doi.org/2040-4468

Schumpeter, J. A. (1934). The Theory of Economic Development: An inquiry into profits, capital, credit, interest and the business cycle. Harvard University Press (Vol. 39).

Shahsiah, N., y Sepahvand, R. (2016). Studyng the mediating role of knowledge and sharing and market orientation to enhance the Intellectual Capital effect on the Organizational Innovation. International Business Management, 10(20), 4776-4782.

Shahtaheri, S. A., y Teymournejad, K. (2020). Effects of knowledge-based, intellectual capital and innovation methods on human resource management: case study of ministry of education, Tehran district 7. International Transaction Journal of Engineering Management \& Applied Sciences \& Technologies, 11(5).

Solís Vázquez, S. Y., Zerón Félix, M., y Sánchez Tovar, Y. (2019). Efectos de la Capacidad de Absorción en la Innovación del sector Industrial en el Norte de México. Nova Scientia, 11(23), 447-472. https://doi.org/10.21640/ns.v11i23.2039

Soo, C., Wei Tian, A., Teo, S. T. T., y Cordery, J. (2017). Intellectual capital-enhancing hr, absortive capacity, and innovation. Human Resource Management, 177(3), 413-417. https://doi.org/10.1002/hrm

Spithoven, A., Clarysse, B., y Knockaert, M. (2011). Building absorptive capacity to organize inbound open innovation in traditional industries. Technovation, 31(1), 10-21.

Subramaniam, M., y Youndt, M. A. (2005). The Influence of Intellectual Capital on the Types of Innovative Capabilities. Academy of Management Journal, 48(3), 450-463.

Sveiby, K. E. (1997). The Intangible Assets Monitor. Journal of Human Resource Costing \& Accounting, 2(1), 73-97. https://doi.org/10.1108/eb029036

Szulanski, G. (1996). Exploring internal stickiness: Impediments to the transfer of best practice within the firm. Strategic Management Journal, (17), 27-43.

Tabachnick, B., y Fidell, L. (2001). Using multivariate statistics. Boston, Massachusetts: Allyn and Bacon.

Teece, D., y Pisano, G. (1994). The dynamic capabilities of firms: An introduction. Industrial and Corporate Change, 3(3), 537-556. https://doi.org/10.1093/icc/3.3.537-a 
La función mediadora de la capacidad absortiva en la relación entre el capital intelectual y la innovación en los institutos de educación superior del sur de Tamaulipas

Tsai, W. (2001). Knowledge transfer in intraorganizational networks: Effects of network position and absorptive capacity on business unit innovation and performance. Academy of Management Journal, 44(5), 996-1004.

Tsou, H. T., Chen, J. S., y Liao, S. W. (Jolie). (2016). Enhancing intellectual capital for e-service innovation. Innovation: Management, Policy and Practice, 18(1), 30-53. https://doi.org/10.1080/14479338.2016.1181527

Varadarajan, R. R., y Jayachandran, S. (1999). Marketing Strategy : An Assessment of the State of the Field and Outlook. Journal of the Academy of Marketing Science.

Wernerfelt, B. (1984). A Resource-Based View of the Firm. Strategic Management Journal, 5, 171-180. https://doi.org/10.2307/2486175

Wold, H. (1985). Partial Least Squares. In S. Kontz \& N. L. Johnson (Eds.), Encyclopedia of Statistical Science (pp. 581-591). New York: Wiley.

Zahra, S., y George, G. (2002). Absortive capacity: a review, reconceptualization, and extension. Academy of Management Review, 27(2), 185-203.

Zhang, M., Lettice, F., y Pawar, K. (2019). Effects of intellectual capital and university knowledge in indigenous innovation: evidence from Indian SMEs. Production Planning and Control, 30(10-12), 799-812. https://doi.org/10.1080/09537287.2019.1582090

Zhang, M., Qi, Y., Wang, Z., Pawar, K. S., y Zhao, X. (2018). How does intellectual capital affect product innovation performance? Evidence from China and India. International Journal of Operations \& Production Management, 1-41. https://doi.org/10.1108/IJOPM-10-2016-0612 